\title{
Prognostic significance of cerebrospinal fluid lymphoblasts at initial presentation with acute lymphoblastic leukemia attending at bangabandhu sheikh mujib medical university, bangladesh
}

\begin{abstract}
Background: Leukemia is characterized by persistent and enormous proliferation of immature white blood cell. Without CNS-directed therapy relapses originating from CNS are up to $75 \%$. The presence of overt CNS disease at initial presentation (cranial nerve palsies) negatively affects the EFS of children with ALL. Early intensive intrathecal and systemic therapy is now more successful in treating and preventing CNS leukemia and reducing the cumulative risk for all relapses with CNS involvement of $4.4 \%$, even in patients whose leukemic blast cells is iatrogenically introduced into the cerebrospinal fluid by traumatic lumbar puncture.
\end{abstract}

Objectives: To determine the prognostic significance of leukemic blasts cell infiltration or traumatic lumbar puncture (TLP) in cerebrospinal fluid (CSF) of newly diagnosed children with acute lymphoblastic leukemia (ALL).

Methods: 546 patients were enrolled based on initial central nervous system (CNS) status: CNS1 (CNS negative, $\mathrm{n}=445)$, CNS2 ( $\leq 5 \mathrm{WBC} / \mathrm{HPF}$ CSF with blasts, $n=35$ ), CNS3 (CNS positive, $n=18$ ), TLP+ (TLP with blasts, $n=38$ ) and TLP- (TLP without blasts, $\mathrm{n}=10$ ). Lumbar punctures (LP) were performed at initial presentation and again at initial intrathecal (IT) therapy. Effect of traumatic lumbar puncture (TLP) were evaluated and followed-up for three years. Two extra doses of intrathecal methotrexate (IT MTX) were given with CNS2 and TLP+ status of patients. Additional IT MTX and cranial irradiation (CI) 24 Gy units were given to children of CNS3 status.

Results: Children with CNS2, CNS3 and TLP+ status found with unfavorable characteristics (high WBC count, raised serum LDH, hepatosplenomegaly, lymphadenopathy, immunological status, response to therapy and risk group etc.). Event free survival (EFS) with one contaminated TLP++ with blasts in CSF was worse than that of without any contamination $(p=0.001)$. EFS with two consecutive contaminated TLP++ in CSF sample found to have significantly poor outcome (5years $\mathrm{EFS}=50 \pm 7 \%$ ). Cox regression analysis showed hazards of adverse effects 2.39 times more common with TLP++ status than those with CNS1 (95\% confidence interval (CI), 1.36-4.20; $\mathrm{p}=0.003$, Cumulative incidence (CI) with TLP ++ status was higher than those with CNS1 (5 years: $32 \pm 10$ and $11 \pm 2$ respectively). TLP+ and CNS3 status had significant prognostic value: risk ratio $(\mathrm{RR})=2.39,95 \% \mathrm{CI}, 1.4$ to 3.6 ; $\mathrm{p}=0.0005 ; \mathrm{TLP}+\mathrm{RR}=1.5,95 \% \mathrm{CI}, 1.02$ to $2.20 ; \mathrm{p}=0.04$. Overall 5 -year EFS were found $75 \%$. EFS with CNS1 and CNS2 status were $82 \%$ and for the TLPstatus was $85 \%$. EFS for CNS3 status was $52 \%$ and suffers from more systemic involvement. CI of relapses with CNS involvement was higher compared with CNS1 status $(0.13$ versus 0.06$)$. Children with TLP+ status had significant

Abbreviations: BSMMU, bangabandhu sheikh mujib medical university; LP, lumber puncture; TLP, traumatic lumber puncture; $\mathrm{TLP}+$, first traumatic lumber positive; TLP++, two consecutive traumatic lumber puncture; TLP-, traumatic lumber puncture negative; CNS, central nervous system; EFS, event free survival; CI, confidence interval; ALL, acute lymphoblastic leukemia; CSF, cerebrospinal fluid; IT MTX, intrathecal methotrexate; WBC, white blood cell; CI, cranial irradiation; IT, intrathecal; CI, cumulative incidence

\section{Introduction}

Leukemia is characterized by persistent and enormous proliferation
Volume 6 Issue 2 - 2017

\author{
Md Golam Hafiz, Chowdhury Yakub Jamal, \\ Afiqul Islam \\ Department of Pediatric Hematology and Oncology,
} Bangabandhu Sheikh Mujib Medical University, Bangladesh

Correspondence: Md Golam Hafiz, Associate Professor, Department of Pediatric Hematology and Oncology, Bangabandhu Sheikh Mujib Medical University, Shahbag, Dhaka, Bangladesh, Email golamhafiz59@yahoo.com

Received: October 26, 2016 | Published: February 13, 2017

decreased EFS $(75 \%, \mathrm{p}=0.003)$ due to increased incidence of CNS relapses. Children with CNS2 had same prognosis as that with CNS1 status, whereas EFS of TLP+ status was inferior to CNS1 status but was superior to CNS3 status $(\mathrm{p}=0.001)$. EFS with two consecutive TLP ++ status contaminated CSF sample was particularly poor.

Conclusion: Contamination of CSF samples with circulating leukemic blasts during diagnostic lumbar puncture, an additional IT MTX is indicated which can prevent adverse effect and outcome of newly diagnosed children with ALL. $\mathrm{CI}$ of isolated hematologic relapse is higher in patients with two consecutive TLP++ compared with that of CNS1 status. For improved treatment outcome every attempt should be made to prevent TLP. So, occurrence of this can adversely affects the quality of life, thus making the need of additional IT MTX therapy.

Keywords: prognostic significance of cerebrospinal fluid lymphoblasts, initial presentation, acute lymphoblastic leukemia, childhood malignancies, diagnostic lumbar puncture

of immature white blood cell in the body. It is responsible for over whelming majority of childhood malignancies. The incidence of leukemia in India and the neighboring countries varies from 0.3 to $1.2 \% .^{1}$ In Bangladesh, though exact incidence of childhood malignancies is still unknown but it appears to be an increase in the cases. One of the difficulties in identifying the problem in Bangladesh is that there is no well-defined birth and death registry covering the whole country. Yet if we take the cognizance of incidence of other countries of this region, we can presume that 5 to 6 thousands new cases should diagnose each year. ${ }^{2}$ Acute lymphoblastic leukemia (ALL) in children is a highly curable childhood malignancy. Now a days, cure rate in the Western countries lies between $70-80 \%{ }^{3}$ 
Rapid diagnosis of acute leukemia is made when a patient develops symptoms and signs which are very important to ensure that the child is in good clinical condition at the time of diagnosis. In countries with limited resources where there is much poverty, high infection rate, lack of public awareness of leukemia and childhood malignancies the symptoms interval is greatly prolonged (usually 4 to 5 weeks) leading to severe deterioration of the patients clinical state before diagnosis is made often with higher initial mortality rates. ${ }^{4}$

For effective treatment of childhood all, it is mandatory to give sufficient CNS directed therapy to treat subclinical or overt CNS leukemia. Without CNS-directed therapy, relapses originating from the CNS are up to $75 \%$ of cases can be expected. ${ }^{5}$ With effective prophylactic CNS directed therapy, such as IT and CI, it is found that up to $80 \%$ of patients in current studies are finally regarded as cured and only about 5\% relapse with involvement of the CNS. ${ }^{6}$ The presence of overt CNS disease at the time of initial presentation as defined by CSF criteria or the presence of cranial nerve palsies negatively affects the EFS of children with all. ${ }^{7-10}$

Early intensive intrathecal and systemic therapy is now more successful in treating and preventing CNS leukemia and reducing the cumulative risk for all relapses with CNS involvement of $4.4 \%$, even in patients whose leukemic blast cells is iatrogenically introduced into the cerebrospinal fluid by traumatic lumbar puncture. ${ }^{11}$ Therefore, cranial irradiation is seldom necessary in contemporary treatment programs. But others have reported neuropsychological deficits in children who received CNS directed therapy consisting solely of approximately 20 intrathecal chemotherapy with methotrexate, hydrocortisone and cytarabine over 3 years. ${ }^{12}$

Inadequate control of CNS leukemia is partly related to the decreased use of cranial irradiation for subclinical disease to avoid the long-term Sequelae of this treatment modality. Indeed, even in patients with CNS leukemia at diagnosis or relapse attempts have been made to reduce the dose of therapeutic cranial irradiation. Thus, most contemporary protocols do not specify cranial irradiation for infants or very young children, even if they present with CNS leukemia. ${ }^{13}$

Gajjar et al., ${ }^{14}$ demonstrates impressively the adverse effect of a traumatic lumbar puncture (defined as more than 10 red blood cells per micro liter of CSF) at the time of diagnosis on treatment outcome of children ALL. The bad prognosis can be predominantly observed in a subgroup of patients with 2 consecutive traumatic lumbar punctures and the detection of leukemic blast cells in the CSF. The authors conclude that the iatrogenic introduction of leukemic blast cells into the CSF may be one of the reasons for the reduced outcome. ${ }^{14}$ There has been a report about the significance of blasts detected in CSF without pleocytosis. Children's Cancer Group reported that children with blasts cell in CSF preparations in the presence of five or fewer WBCs per micro liter of CSF are not at greater risk for CNS or other relapse compared with CNS-negative patients. ${ }^{15,16}$ In contrast, investigators from St. Jude Children's Research Hospital have shown that $\mathrm{CNS} 2$ status $(<5 \mathrm{WBC} / \mathrm{L}$ CSF with blasts) resulted in a higher risk of relapse and would thus require more intensive intrathecal therapy. ${ }^{14,17}$

In addition, most of the studies looking at outcome in children within CNS status subgroups to date had not evaluated that particular subset of patients with traumatic lumbar punctures at diagnosis. An up-to-date data demonstrating that initial traumatic lumbar punctures (TLPs) combined with the presence of blasts negatively affects treatment outcome. ${ }^{14}$ The presence or absence of CNS leukemia at diagnosis has prognostic significant value. Patients who have a non traumatic diagnostic lumbar puncture may be distributed into one of three categories according to the number of $\mathrm{WBC} / \mu \mathrm{L}$ and the presence or absence of blasts on cytospin as follows:

I. CNS1: Cerebrospinal fluid (CSF) that is cytospin negative for blasts regardless of WBC count.

II. CNS2: CSF with fewer than $5 \mathrm{WBC} / \mu \mathrm{L}$ and cytospin positive for blasts.

III. CNS3 (CNS disease): CSF with 5 or more $\mathrm{WBC} / \mu \mathrm{L}$ and cytospin positive for blasts.

Children with ALL who present with CNS disease (CNS3) at diagnosis are at a higher risk of treatment failure (both within the CNS and systemic involvement) than are the children who are classified as CNS1 or CNS2. ${ }^{19}$ Some studies have reported increased risk of CNS relapse and/or inferior EFS in CNS2 patients, compared with CNS1 children, ${ }^{19,17}$ while others have not such involvement. ${ }^{18,15,20}$

A traumatic lumbar puncture ( $\geq 10$ erythrocytes $/ \mu \mathrm{L})$ that includes blasts at diagnosis has also been associated with increased risk of CNS relapse and overall poorer outcome in some studies, ${ }^{14,18,21}$ but not in others patients. ${ }^{19,20}$ Patients with CNS2, CNS3, or traumatic lumbar puncture have a higher frequency of unfavorable prognostic characteristics than do those with CNS1,including significantly higher WBC counts at diagnosis, the patients with older age (more than 10years) at diagnosis have an increased frequency of the T-cell ALL phenotype and MLL gene rearrangements. ${ }^{18,20,21}$ Some clinical trial groups have approached CNS2 and traumatic lumbar puncture by utilizing more intensive therapy, primarily additional doses of intrathecal therapy during induction of remission..$^{18,20,22}$ Other groups have not changed therapy based on CNS2 status. ${ }^{16,19}$ A child with a traumatic lumbar puncture (with blasts) should be treated as CNS3, the COG uses an algorithm relating the WBC count and red blood cell counts in the spinal fluid and the peripheral blood film. ${ }^{23}$

\section{Materials and Methods}

\section{CNS status}

From January 2009 to December 2014, 546 children with newly diagnosed ALL were enrolled in the study protocol: ALL BerlinFrankfurt-Münster-95 (ALL-BFM 95) at Pediatric Hematology and Oncology Department of Bangabandhu Sheikh Mujib Medical University, Shahbag, Dhaka, Bangladesh. Informed written consent was obtained from the guardians of the patients. The patients with a minimum follow-up of 3years were included in this prospective study. All the patients underwent LP at initial diagnostic bone marrow aspiration and again on the first day of induction of remission therapy (usually within 24 to 48 hours) after diagnostic procedures when IT MTx was to be started. CNS status was defined as follows: CNS1 (puncture non traumatic without leukemic blasts cell after cytocentrifugation), CNS2 (puncture nontraumatic, $<5 \mathrm{WBC} / \mathrm{L} \mathrm{CSF}$ with identifiable leukemic blasts cell), CNS3 (puncture nontraumatic, $\geq$ $5 \mathrm{WBC} / \mathrm{L}$ CSF with identifiable leukemic blasts cell), TLP+(with leukemic blast cells after cytocentrifugation) and TLP- (puncture traumatic, 10 red blood cells per micro liter) with no leukemic blast cells after cytocentrifugation). CNS status of children for whom 2 consecutive TLPs contained leukemic blast cells was classified as TLP++. CNS3 definition was derived from Mastrangelo et al., ${ }^{24} \mathrm{~A}$ TLP was defined as 10 or more erythrocytes per microliter CSF or macroscopically contaminated CSF. In addition to the CNS3 group as defined above, children with a cerebral mass or children with cranial nerve palsy in combination with blasts cell after cytocentrifugation were considered to have CNS3 disease at initial presentation. 


\section{Supportive care}

Children with ALL who were included in the study had complete blood count, liver function, renal function test, serum $\mathrm{LDH}$, chest $\mathrm{X}$-Ray, blood group, coagulation profile, hepatitis B screening and bone marrow aspiration at initial presentation when attended at the out-patient department. There was no fixed policy regarding blood transfusion prior to the procedures for investigation. Generally, child received blood transfusion or platelet transfusions if there was/were signs of overt bleeding or subcutaneous bleeding manifestation in the form of petechae, purpura or echymoses or impending intracranial haemoharrge. The presence of coagulopathy at the time of diagnosis has no significant value if there is any consequence in the childhood ALL, in such case no therapeutic intervention is initiated for this finding. ${ }^{25}$ The bone marrow aspiration were performed by the expert physician and assisted by the nurses and resident students of the department.

\section{Treatment}

Patients were treated according to the protocols of ALL-BFM 95, where patients were stratified according to age, initial WBC count, response to prednisone on the day 8 of therapy, molecular rearrangements $\mathrm{t}(9 ; 22) ; \mathrm{t}(4 ; 11)$ and into risk groups: standard risk (SR), medium-risk (MR), and high-risk (HR). The children with SR and MR group treated with induction therapy (prednisone, vincristine, daunorubicin, L-asparaginase, Intra-thecal (methotrexate, cytarabine, hydrocortisone) for 8 weeks consisted of an eight-drug induction of remission ${ }^{26,27}$ then, consolidation with three times highdose methotrexate (HDMTX:2.5-3.0 gm/ $\mathrm{m}^{2}$ ) and an eight-drug reintensification, followed by the standard maintenance therapy. HR patients were treated with a five-drug induction therapy, followed by six intensive multiagent blocks and the reintensification as SR and MR children. CNS-directed therapy consisted of 12 doses of IT MTX for both SR and MR patients, whereas, HR children were received six doses of Triple Intrathecal Therapy (TIT) with MTx, cytarabine and hydrocortisone. Prophylactic CI (18 Gy units) was given after reintensification only to those children with HR groups. The patients with CNS2 status and TLP+ disease received two additional doses of IT MTx during the period of induction of remission. Some TLP+ children (05) were treated as having CNS3 disease although CSF findings were not consistent with the CNS3 definition as described earlier. The children with CNS3 status received four to five additional doses of IT MTx therapy and therapeutic CI with 24 Gy units. When the child was on maintenance therapy IT MTx therapy was given every three month interval having a complete blood count, liver and renal function test during the whole maintenance period of time for 2 to 3years.

\section{Design and analysis}

EFS are defined as the time from initial presentation at diagnosis until the date of the first adverse event (relapse, death for any reason or the development of a second malignancy) or, if no such event occurred, until the date of last follow-up visit. Patients who did not achieve complete remission within 45 days of chemotherapy from initial presentation were considered no EFS. Duration of CNS remission was estimated from the time of initial complete remission to the time of isolated or combined CNS relapse for children who had such a relapse or to the last follow-up time for those whose disease remained in CR. All non-CNS relapses, second malignancies and deaths in $\mathrm{CR}$ were considered competing risks for developing a CNS relapse and were analyzed accordingly. Duration of hematologic remission was measured from the time of initial complete remission to the time of isolated hematological relapse for patients who had such a relapse or to the last follow-up time for those whose disease remained in CR. All other types of relapse, second malignancies and deaths in $\mathrm{CR}$ were considered competing risks for developing an isolated hematologic relapse and the results were analyzed accordingly.

Fisher exact test and the exact chi-square $(\chi 2)$ test were used to test for associations between 2 categorical variables. The distributions of EFS and continuous complete remission were estimated by the methods of Kaplan and Meier with Standard error (SE) according to Greenwood and were compared using the log-rank test. Differences in the distribution of variables among patient subsets were analyzed using the student's' test for categorized variables and the Wilcoxon rank sum test had done for continuous variables. The prognostic relevance of different CNS groups compared with CNS-negative patients measured by Cox regression analysis with known prognostic factors as co-variables. All estimation of outcome was reported as \pm (1SE). All analyses of outcome were stratified by study number and by $\mathrm{NCI} /$ Rome risk criteria, ${ }^{7}$ which are defined as follows: - for children with B-lineage ALL: standard risk (WBC at diagnosis $<50 \times 109 / \mathrm{L}$ and age at diagnosis $\geq 1$ and $<10$ years) and high risk (WBC at diagnosis $\geq 50 \times 109 / \mathrm{L}$ or age at diagnosis $\geq 10$ years. For the purpose of this study, the children with T-lineage ALL and infants younger than 1 year of age were also considered as high risk. Cox proportional hazards regression mode. ${ }^{28}$ was used to evaluate the significance of having two consecutive traumatic taps (TLP++) with blasts with respect to EFS while controlling for other known adverse risk factors. ' p'values reported are two-sided and all analyses were conducted with the use of SPSS 17.0.The 'p'value of less 0.05 was considered as a test of significance.

\section{Result}

Total 546 patients were rearranged into each of CNS status: CNS 1 (445), CNS 2(35), CNS 3 (18), TLP+ (38) and TLP-(10). 81.50\% of patients were found CNS1 status and $3.29 \%$ were with CNS3 status. The remaining $15.21 \%$ patients were divided among the other status: CNS2 status (6.41\%), TLP+ status $(6.95 \%)$ and TLP- status (1.83\%). Out of eighteen patients with CNS3 status, three had CNS leukemia by the demonstration of a cerebral/meningeal mass only and five had CNS3 status based on the presence of blasts cells without CSF pleocytosis along with cranial nerve palsy. It is observed that children with blasts cell at presentation in CSF were significantly associated with hepatosplenomegaly $(\mathrm{p}<0.01 ;$ Table 1$)$. The children with TLP with blasts cell at presentation was significantly associated with generalized lymphadenopathy $(p<0.04$; Table 1$)$. Statistically significant value also found with TLP with blasts cell in CSF at presentation with meadiastinal mass $(p<0.01$; Table 1$)$. The characteristics of children among different CNS status observed with increased percentage of unwanted association in CNS2, CNS3 and TLP+ status (Table 1). CNS2, CNS3 and TLP+ groups had significantly higher $\mathrm{WBC}$ counts at presentation in comparison to CNS1 group (Wilcoxon rank test, $\mathrm{p}=0.0001$ ) and it was significantly less in SR children and more in HR children (Chai2 test, $\mathrm{p}<0.01$ ). A significant number of children $(80 \%)$ with CNS1 status found between 1 to 9 years of age $(\mathrm{p}=0.01)$. The patients with $\mathrm{CNS} 3$ and TLP+ status were found with high serum LDH $(>1000),(p=0.01$; Table 1). About $55 \%$ of children with CNS3 status were found with significant generalized lymphadenopathy compared with CNS1 status $(\mathrm{p}=0.04)$. A statistically significant difference in platelet count could not be elicited (median platelet count in TLP+ children: 68,500/L; median platelet count in CNS1 patients: 75,000/L; (Wilcoxon rank test, $\mathrm{p}=0.131$ ). In $\mathrm{CNS} 2$ status there were significantly large number of children with T-ALL and pro-B-ALL. The CNS3 status of children 
found with significantly more with T-lineage ALL (Chai2 test, $\mathrm{p}<0.01)$. CNS-directed therapy in children with ALL in different groups showed (Table 2) that 20 children received cranial irradiation, 337 patients of CNS1 status had received chemotherapy, 55 patients received radiotherapy in CNS1 group of children, none of the children received radiotherapy in TLP- group. Thirty children in CNS1 status had received additional ITMTx, 31 children in TLP + group but only two patients in TLP- status. In CNS2 group 27 received additional intrathecal methotrexate and one patient received radiotherapy. TLP group received 31 additional intrathecal methotrexate and 1 received radiotherapy. In TLP- group received no radiotherapy but 2 children received additional intrathecal methotrexate. Regarding the CNS directed therapy, when compared the TLP+ children (B-precursor and T-ALL) treated as CNS negative $(\mathrm{n}=04)$ with those who received two additional doses of IT MTx $(n=31)$, no statistical significant difference could be found, although there was a tendency toward higher EFS with additional IT MTx $(67 \% \pm 5 \%)$ versus $(58 \% \pm 7 \%),(\mathrm{p}=0.59)$. The observation was similar for CNS2 status of children, EFS ( $65 \% \pm 8 \%$ ) for the children treated as CNS- $(n=07)$ treated as negative with those who received two additional doses of IT MTx $(n=27)$, no statistical significance difference were found rather than a tendency toward higher EFS with additional IT MTx $(77 \% \pm 5 \%)$ versus $(71 \% \pm 6 \%)$ $(\mathrm{p}=0.45) .5$-years EFS found $( \pm 1 \mathrm{SE})$ for patients in each group were as follows: CNS $1(82 \pm 2 \%)$, CNS $2(82 \pm 3 \%)$, CNS $3(52 \pm 6 \%)$, TLP+ $(75 \pm 5 \%)$ and TLP- $(85 \pm 4 \%$; Figure 1$)$. The EFS for CNS1, CNS2 and TLP- status were almost identical at $82 \%$. When compared with the children of CNS1, overall prognosis of the children with TLP+ was significantly worse $(75 \%, \mathrm{p}=0.004)$. 5years EFS for the children with CNS3 found $52 \%$, had significant worst prognosis $(\mathrm{p}=0.0001)$. We found that twenty patients with two consecutive TLPs with blast cells (TLP++) in the CSF which had significantly worse outcome than those of the children with CNS1 status even after stratifying for treatment protocol and NCI/Rome risk criteria $(\mathrm{p}=0.001$; Figure 2). The treatment outcome of these children with TLP++ was comparable to that of children with overt CNS disease (CNS 3 status) $(\mathrm{p}=0.94)$. Cox regression analysis stratified by treatment protocol, the hazard of adverse events was found to be 2.39 times more likely for the children with TLP++ than for those with CNS 1 status $(95 \%$ confidence interval, 1.36-4.20 ( $\mathrm{p}=0.003$; Table 4$)$. The effect of having two consecutive TLPs with blast cells on the cumulative incidence of developing either isolated or combined CNS relapse. Our findings showed that the CI for any relapse in children with CNS1 status was 0.20 , CI for an isolated or combined relapse was 0.06 and for other relapses (bone marrow, testes and other) was 0.16 (Table 3 ). It has also found among TLP- status of children. There was higher proportion of relapse with CNS involvement among the CNS2 group of children, CI 0.13. The children with CNS3 status were observed with highest overall relapse rate (CI 0.35). In TLP+ status, we found an increase in CI for all relapses (0.25), CI for relapses with CNS involvement (0.05) which was comparable to CNS2 status of children but the CI for other relapses $(0.15)$ was nearer to CNS1 status. We also observed that CNS3 status have the highest overall rate of relapse $(0.27)$. The effect of having two consecutive TLP++ with blasts cell found cumulative incidence (CI) with TLP++ was higher than that of those with CNS1 status (5-years found: $16 \pm 8 \%$ and $4 \pm 1 \%$, respectively; $\mathrm{NCI} /$ Rome risk criteria; $\mathrm{p}=0.094)$. CI of isolated hematological relapse was higher with TLP++ status compared with that of CNS1 status (5-years found: $32 \pm 10 \%$ and $11 \pm 2 \%$, respectively; NCI/Rome risk criteria; ( $\mathrm{p}=0.019$; Figure 2$)$.

Table I Characteristics of Patients According to Clinical Findings and CNS Status

\begin{tabular}{|c|c|c|c|c|c|c|}
\hline Parameters & All (\%) & CNSI(\%) & CNS2(\%) & CNS3(\%) & TLP+(\%) & TLP- (\%) \\
\hline All & $546(100)$ & $445(82)$ & $35(6)$ & $18(3)$ & $38(7)$ & $10(2)$ \\
\hline \multicolumn{7}{|l|}{ Sex } \\
\hline Male & $315(58)$ & $255(57)$ & $21(60)$ & 13(72)* & $23(6 I)$ & $7(70)$ \\
\hline Female & $23 I(42)$ & $190(43)$ & $14(40)$ & $05(28) *$ & $15(39)$ & $3(30)$ \\
\hline \multicolumn{7}{|l|}{ Age } \\
\hline$<1$ year & $20(3)$ & $10(2)$ & $2(6)$ & $02(1 \mathrm{I})$ & $02(5)$ & $01(10)$ \\
\hline I to 9 year & $435(80)$ & $360(8 I)$ & $25(7 I)$ & $12(67)$ & $25(66) *$ & $7(70)$ \\
\hline$>10$ year & $91(17)$ & $75(17)$ & $08(23)$ & $04(22)$ & II (29)* & $02(20)$ \\
\hline \multicolumn{7}{|l|}{ WBCX 109} \\
\hline$<50,000$ & $372(68)$ & $310(70)$ & $16(46)^{*}$ & $06(33) *$ & $17(45)^{*}$ & $07(70)$ \\
\hline $50,000-1,00000$ & $119(22)$ & $110(25)$ & $13(37) *$ & $04(22) *$ & $18(47)^{*}$ & $02(20)$ \\
\hline$>1,00000$ & $55(10)$ & $25(05)$ & $06(17)^{*}$ & $08(45)^{*}$ & 03(08)* & $0 \mathrm{I}(10)$ \\
\hline \multicolumn{7}{|l|}{ Serum LDH } \\
\hline$<400$ IU & $6 I(11)$ & $45(10)$ & $02(06)$ & $02(11)$ & $03(08)$ & $02(20)$ \\
\hline 400 to $1000 \mathrm{IU}$ & $360(66)$ & $225(5 I)$ & $21(60)$ & $07(39) *$ & $21(55)^{*}$ & $05(50)$ \\
\hline$>1000 \mathrm{IU}$ & $125(23)$ & $175(39)$ & $12(34)$ & $09(50) *$ & $14(37)^{*}$ & $03(30)$ \\
\hline \multicolumn{7}{|l|}{ Hepatomegaly } \\
\hline$<5 \mathrm{~cm}$ & $4 I(07)$ & $75(17)$ & 03(9) & $03(17)$ & 04(II) & $01(10)$ \\
\hline 5 to $10 \mathrm{~cm}$ & $430(78)$ & $290(65)$ & $27(77)$ & $\mathrm{II}(6 \mathrm{I})$ & $24(63)^{*}$ & $06(60)$ \\
\hline$>10 \mathrm{~cm}$ & $75(13)$ & $80(18)$ & $05(14)$ & $04(22)^{*}$ & $10(26)^{*}$ & $03(30)$ \\
\hline \multicolumn{7}{|l|}{ Splenomegaly } \\
\hline$<5 \mathrm{~cm}$ & $33(06)$ & $5 I(12)$ & $06(17)$ & $05(28)$ & $06(16)$ & $0 I(10)$ \\
\hline 5 to $10 \mathrm{~cm}$ & $336(62)$ & $299(67)$ & 17(49) & $09(50)^{*}$ & $2 \mathrm{I}(55)^{*}$ & $08(80)$ \\
\hline$>10 \mathrm{~cm}$ & 177(32) & $95(2 I)$ & $12(34)$ & $04(22)$ & II(29) & $01(10)$ \\
\hline \multicolumn{7}{|l|}{ Lymphadenopathy } \\
\hline$<2$ group involved & $61(11)$ & $65(15)$ & $06(17)$ & $03(17)$ & $06(16)^{*}$ & $03(30)$ \\
\hline 2 to 4 groups involved & $380(70)$ & $245(55)$ & $18(52)$ & $10(56)^{*}$ & $23(60)$ & $04(40)$ \\
\hline$>4$ groups involved & $105(19)$ & $135(30)$ & $11(31)^{*}$ & $05(27)$ & $09(24)$ & $03(30)$ \\
\hline \multicolumn{7}{|l|}{ Immunology } \\
\hline Pro-B & $43(08)$ & $27(06)$ & $06(17)^{*}$ & $03(17)$ & $08(2 I)$ & $02(20)$ \\
\hline Pre-B/common & $42 I(77)$ & $298(67)$ & $17(49)$ & $07(38)$ & $17(45)$ & $06(60)$ \\
\hline T-ALL & $82(15)$ & $120(27)$ & $12(34)^{*}$ & $08(45)$ & $13(34)$ & $02(20)$ \\
\hline
\end{tabular}


Table Continued...

\begin{tabular}{lllllll}
\hline Parameters & All (\%) & CNSI(\%) & CNS2(\%) & CNS3(\%) & TLP+(\%) & TLP- (\%) \\
\hline Response & & & & & & \\
Good & $390(7 I)$ & $4 I I(92)$ & $29(83)$ & $12(67)^{*}$ & $33(87)$ & $09(90)$ \\
Poor & $156(29)$ & $34(08)$ & $06(17)$ & $06(33)^{*}$ & $05(13)$ & $01(10)$ \\
Risk Group & & & & & \\
SR & $180(33)$ & $175(39)$ & $07(20)^{*}$ & $02(I I)^{*}$ & $07(18)^{*}$ & $03(30)$ \\
MR & $295(54)$ & $230(52)$ & $22(63)^{*}$ & $10(56)^{*}$ & $26(69)^{*}$ & $05(50)$ \\
HR & $7 I(13)$ & $40(09)$ & $06(17)^{*}$ & $06(33)^{*}$ & $05(I 3)^{*}$ & $02(20)$ \\
\hline
\end{tabular}

Note: Percentages are in parentheses. In the first column, percentages refer to whole group; in the other column, percentages are calculated within the CNS status group. Statistically significant differences compared with the CNSI group, are marked with an asterisk. (Categorized variables done with $\square^{2}$ test, continuous variable (WBC count, Serum LDH and other) done with Wilcoxon rank sum test.

Table 2 CNS Directed Therapy to the children Based on CNS Status

\begin{tabular}{|c|c|c|c|c|c|c|}
\hline $\begin{array}{l}\text { Treatment (Chemotherapy } \\
\text { and others) }\end{array}$ & $\begin{array}{l}\text { All }(n=546) \\
(\%)\end{array}$ & $\begin{array}{l}\text { CNSI } \\
(n=445)(\%)\end{array}$ & $\begin{array}{l}\text { CNS2 } \\
(n=35)(\%)\end{array}$ & $\begin{array}{l}\text { CNS3 } \\
(n=18)(\%)\end{array}$ & $\begin{array}{l}\text { TLP+ } \\
(n=38)(\%)\end{array}$ & $\begin{array}{l}\text { TLP-(n=10) } \\
(\%)\end{array}$ \\
\hline Therapy, CNS negative & $375(69)$ & $337(76)$ & $07(20)$ & $00(00)$ & $04(10)$ & $08(80)$ \\
\hline Additional Intrathecal MTX & $117(21)$ & $30(07)$ & $27(77)$ & $04(22)$ & $3 \mid(82)$ & $02(20)$ \\
\hline Therapy, CNS positive & $34(06)$ & $23(05)$ & $00(00)$ & $13(72)$ & $02(05)$ & $00(00)$ \\
\hline Radiotherapy & $20(04)$ & $55(12)$ & $0 \mathrm{OI}(03)$ & $01(06)$ & $0 \mathrm{OI}(03)$ & $00(00)$ \\
\hline
\end{tabular}

Note: $T L P=$ Traumatic lumbar puncture, IT MTX= Intrathecal methotrexate, $C N S=$ Central nervous system.

Table 3 Distribution of Types of Relapses Based on CNS Status of the Children

\begin{tabular}{|c|c|c|c|c|c|c|}
\hline Distribution of Events & $\begin{array}{l}\text { All } \\
(n=546)\end{array}$ & $\begin{array}{l}\text { CNSI } \\
(n=445)\end{array}$ & $\begin{array}{l}\text { CNS2 } \\
(n=35)\end{array}$ & $\begin{array}{l}\text { CNS3 } \\
(n=18)\end{array}$ & $\begin{array}{l}\text { TLP+ } \\
(n=38)\end{array}$ & $\begin{array}{l}\text { TLP. } \\
(n=\mid 0)\end{array}$ \\
\hline All relapses & $287(0.17)$ & $227(0.17)$ & $19(0.17)$ & $\mathrm{II}(0.35)$ & $24(0.20)$ & $06(0.12)$ \\
\hline Relapses with CNS involvement & $89(0.043)$ & $48(0.035)$ & $24(0.10) *$ & $09(0.13)^{*}$ & $06(0.08) *$ & $02(0.02)$ \\
\hline Isolated CNS relapse & $52(0.53)$ & $3 I(0.45)$ & $09(0.13)$ & $07(0.05)$ & $03(0.04)$ & $02(0.19)$ \\
\hline Bone marrow and CNS relapse & $38(0.03)$ & $26(0.02)$ & $04(0.08)$ & $04(0.01)$ & $03(0.03)$ & $0 I(0.03)$ \\
\hline Other relapse & $17 \mid(0.13 \mid)$ & $139(0.134)$ & $12(0.07)$ & $09(0.22)$ & $08(0.12)$ & $03(0.10)$ \\
\hline Other events(death during CCR, second malignancy, LFU) & $85(0.03)$ & $53(0.03)$ & $09(0.02)$ & $07(0.10)$ & $10(0.05)$ & $06(0.06)$ \\
\hline
\end{tabular}

Note: Cumulative incidences are showed in parentheses. Statistically significant differences compared with CNSI patients are marked with an asterisk.

$\mathrm{BM}=$ bone marrow, $\mathrm{CCR}=$ continuous complete remission, $\mathrm{LFU}=$ lost to follow up in $\mathrm{CCR}, \mathrm{CNS}=$ central nervous system.

Table 4 Prognostic significance of two consecutive positive traumatic lumber punctures (TLP++) in children with CNSI Status or TLP++

\begin{tabular}{llll}
\hline Adverse Effect & Hazard Ratio(HR) & Confidant Interval $\mathbf{( 9 5 \% ~ C l )}$ & P value \\
\hline TLP++ & 2.39 & $1.36-4.20$ & 0.003 \\
NCl high-risk group & 2.04 & $1.29-3.24$ & 0.002 \\
DNA index<1.16>I.6 & 1.53 & $0.83-2.80$ & 0.17 \\
T-cell ALL & 1.51 & $0.85-2.67$ & 0.16 \\
\hline
\end{tabular}

Note: CNS: Central Nervous System; Cl: Confidence Interval; NCl: National Cancer Institute;ALL:Acute Lymphoblastic Leukemia.

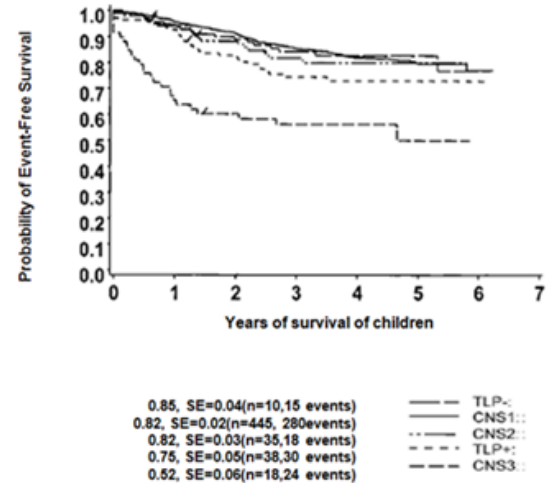

Figure I Event free survival (EFS) of CNS status those who are entitled in the study with a minimum follow-up of three years. EFS of TLP+ children is significantly inferior to CNSI status $(p=0.003)$ but superior to CNS3 status $(\mathrm{p}=0.00 \mathrm{I})$.

EFS of Children Based on the Different CNS Status Group

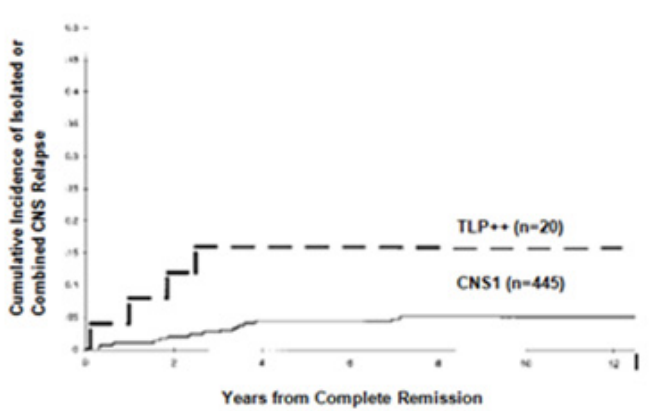

Figure 2 Cumulative incidence of an Isolated or combined CNS relapse for children with CNSI status and TLP++ status.

\section{Discussion}

Gilchrist and coworkers ${ }^{29}$ evaluated the prognostic influence of blast cells in cerebrospinal fluid among patients with low leukocyte 
counts. Their analyses showed no association between this factor and relapse confined to the central nervous system. However, their exclusion of patients at high risk, the use of early cranial irradiation in half their patients, and differences in systemic chemotherapy among their patients could explain the differences between our study and theirs findings. ${ }^{30}$

The distribution of children within the CNS status group is similar to the distribution reported by Gajjar et al., ${ }^{14}$ although group sizes of CNS2, TLP+, TLP- children are smaller in our study. Because of the smaller number of patients and single center study, the group size of CNS2 $(6 \%)$, CNS3 (3\%), TLP+ $(6 \%)$ and TLP-(1\%). Gajjar et al. ${ }^{14}$ reported CNS2 (5\%), CNS3 (3\%), TLP+ (7\%) and TLP- $(6 \%)$ which might have some impact on actual findings in our study. In this prospective study, we found that traumatic lumber punctures with blasts cell at initial presentation in children with ALL negatively affect in the outcome. The children who had traumatic lumber puncture at diagnosis, the risk of treatment failure was 2.57 times more than those who did not have blasts cell in the cerebrospinal fluid. Our observation are consistent with the report of Gajjar et al., ${ }^{14}$ who demonstrates impressively the adverse effect of a traumatic lumbar puncture (defined as more than 10 red blood cells per microliter of cerebrospinal fluid $\mathrm{CSF}$ ) at the time of diagnosis on treatment outcome of children with acute lymphoblastic leukemia (ALL). The inferior prognosis can be predominantly observed in a subgroup of patients with 2 consecutive traumatic lumbar punctures and the detection of leukemic blast cells in the CSF. The authors conclude that the iatrogenic introduction of leukemic blast cells into the CSF may be one of the reasons for the reduced outcome.

The presence of leukemic blast cells in the CSF at the time of diagnosis generally indicates a poor outcome. Leukemic cells in the CSF arise from the cranial arachnoid tissue. ${ }^{31-34}$ The circulating leukemic cells reach the walls of the superficial veins where they extend through the superficial arachnoid into the arachnoid surrounding the arteries, veins, arterioles and venules as they course into and through the brain. With increasing mass the leukemic cells reduce the caliber of the vessels producing cerebral hypo perfusion. Eventually, the leukemic cells can move out of the arachnoid trabeculae into the CSF resulting in iatrogenic leukemic meningitis. ${ }^{35}$ An increasing number of leukemic cells in the CSF reflect either more aggressive leukemia or more advanced disease. The presence of one leukemic blast cell per microliter of CSF corresponds to approximately 105 leukemic cells in the entire CSF compartment. TLP at the time of diagnosis, when most patients have circulating blast cells may be another way of introducing leukemic blasts from the systemic circulation into the CSF. ${ }^{36-38} \mathrm{As}$ expected, from this study, TLP with blast cells was associated with a higher number of total leukocyte counts at presentation.

We found that the children with five or fewer WBCs per microliter CSF with blasts (CNS2) at presentation have the same prognosis regarding EFS as CNS negative children if treated with two additional doses of IT MTX (EFS for both groups, 82\%; SE 2\% and 3\%, respectively). TLP negative children had the same overall outcome (EFS 84\%) as CNS1 and CNS2 groups. TLP positive children, however, were found to have an EFS of $75 \%$, which is inferior to that of CNS1 patients ( $p=0.003$ ) but superior to CNS3 patients (EFS 52\%, $\mathrm{p}=0.001)$.

The CNS2 group contains a higher percentage of children with unfavorable prognostic parameters. There are fewer number of SR and larger number of HR children, T and pro-B immunology are more and the children are presented with higher number of WBC count at initial presentation. We found in this group of children with infiltration of leukemic cell resulting poor prognosis. We found that survival of children with CNS2 status was identical to the EFS in CNS1 and TLP-groups $(82 \%, 82 \%$ and $84 \%$ respectively). It is reported by Pui et al., ${ }^{11}$ that early intensification of IT MTx therapy resulted in a reduced cumulative risk for all relapses with CNS involvement of 4.4\%. Compared with CNS1 patients of whom the majorities are suffering systemic relapses, the relapses among CNS2 patients seem to be more equally divided between those with CNS involvement and all other relapses. This distribution $(50 \%$ relapses with CNS involvement, $50 \%$ bone marrow and other relapses) was also found by Children's Cancer Group study. ${ }^{16}$ We found EFS of $82 \%$ is strikingly different than the experience from St. Jude's Hospital, where CNS2 patients with regular CNS directed therapy had a survival of only55\% ${ }^{14}$ or $53 \%{ }^{17}$ respectively. This result is remarkable, as the number of IT MTx therapy applications (eight to twelve times for lower-risk and twelve to twenty times for higher-risk patients). The percentage of patients receiving cranial irradiation is higher in the St. Jude trial $21 \%$ but in our study it is less. One explanation for this difference might be the fact that, in the St. Jude trails. IT MTx therapy was generally not given at the time of the diagnostic lumber puncture, but 24 to 48 hours later and the second IT MTx therapy was not given until 3 weeks later, whereas in our study, the second IT MTx was always instilled on the day 8 . One could also argue that systemic treatment has improved with time.

There is a report by Bleyer ${ }^{29}$ that certain types of leukemia penetrate the CNS with a higher rate (T-cell leukemias) and other factors (blasts cell and platelet count, patient's age or maturity of the blood-brain barrier) are factors that influence the infiltration of leukemic cells into the CNS.

We did not find any significant value in T-ALL or lower platelet counts among the TLP+ children, but we found a significant number of children had higher initial WBC counts, hepatosplenomegaly, lymphadenopathy, large meadiastinal mass and high serum LDH compared with CNS1 patients. Cox regression analysis reported that TLP+ status maintain prognostic significance, indicating that the higher incidence of unfavorable patient characteristics cannot account for the observed difference in EFS. Also, when comparing TLP+ patients with those in the CNS2 group, which has a good prognosis, TLP+ patients do not seem to have more advanced disease. CNS2 group comprises more children with initial hyperleukocytosis, immunophenotype and prednisone poor-response or high-risk disease than does the TLP+ group. The poor EFS in the TLP+ group could be that, among TLP + status of children, there are hidden CNS3 patients who are simply not identified at the time of initial diagnosis.

The outcome for the TLP+ group also differs from the results reported by Gajjar et al., ${ }^{14}$ although the variation is not as pronounced as for the CNS2 group of children. The reason for this may be the time interval between diagnostic and TLP and the interval to the second instillation of IT MTx therapy. TLP+ children have inferior outcome when compared with CNS2 children because of an iatrogenic introduction of leukemic blasts cell into CSF. But it is not easy to understand why a single iatrogenic introduction of blasts cell should cause a difference in overall outcome of the patients, when patients with "minimal meningeal leukemia," as in the CNS2 group, can be handled quite easily with two additional applications of IT MTX. Another hypothesis to explain the inferior outcome in TLP+ patients could be more advanced disease, for example, with more perivenular or parameningeal leukemic infiltrates or a biologically different disease, permitting migration of leukemic blasts cell more easily. Bleyer ${ }^{29}$ reported that certain types of leukemia penetrate the CNS with a higher rate (eg, T-cell leukemias) and that other factors 
such as blast and platelet count, patient's age or maturity of the bloodbrain barrier are factors that influence ingress of leukemic cells into the CNS. We did not find significantly more T-ALL or lower platelet counts among the TLP+ patients, but we did find a significant number of patients had higher initial WBC counts compared with CNS1 patients. But by Cox regression analysis, TLP+ status maintains prognostic significance, indicating that the higher incidence of unfavorable patient characteristics cannot account for the observed difference in EFS. Also, when comparing TLP+ patients with those in the CNS2 group, which has a good prognosis, TLP+ patients do not seem to have more advanced disease. On the contrary, the CNS2 group comprises more patients with initial hyperleukocytosis, $\mathrm{T}$ immunophenotype and prednisone poor-response or high-risk disease than does the TLP+ group. A third explanation for the inferior EFS in the TLP+ group could be that, among TLP $b$ patients, there are hidden CNS3 patients who are simply not identified at the time of initial diagnosis. Because the other possible explanations for the inferior outcome in TLP b patients seem unlikely, we favor the latter possibility-unidentified CNS3 patients as the most likely explanation for the observed difference in outcome. Cranial RT might lead to significant toxicity or second malignancies in patients who otherwise have a good prognosis. There was no statistically significant difference in TLb patients treated as CNS negative (EFS 66\%) versus those treated with additional IT MTX (EFS 77\%). However, it is better to continue the treatment of CNS2 status and TLP+ status patients with two additional doses of IT MTX.

\section{Conclusion}

We conclude that CNS2 children have good prognosis (EFS 82\%) that does not require any intensification of CNS-directed therapy. TLP+ children have inferior prognosis compared with CNS1 group (75\% versus $82 \%$ ) but has superior EFS compared with the CNS3 status (52\%). For improved treatment outcome every attempt should be made to prevent TLP. Occurrence of this adversely affects the quality of life, making the need of additional IT MTx therapy. CI of isolated hematologic relapse is higher in patients with two consecutive TLP++ compared with that of CNS1 status. With appropriate testament schedule intensive CNS-directed therapy is not mandatory.

\section{Future suggestions}

Diagnostic lumbar puncture should be performed by an experienced physician at initial presentation when there are higher counts with blasts. Hence, we should implement several steps to decrease the frequency and consequence of TLP. The procedure is now routinely performed by experienced clinicians with short-acting general anesthesia (midazolam, $0.05 \mathrm{mg} / \mathrm{kg}$ body wt. i.v). ${ }^{39-41}$ Over and above, IT MTx therapy is now given with the first diagnostic LP performed after the diagnosis of leukemia has been confirmed. In the event of a TLP, this approach may reduce the likelihood that contaminated leukemic cells that seed in the meninges.

\section{Ethical consideration}

The Institutional ethical committee and review board of BSMMU approved the treatment protocols and signed informed consent was obtained from the patients, their parents or their guardians as appropriate.

\section{Limitations of the study}

This is a single center study. So, further multicenter studies with large sample are necessary for the detection of CNS status, survival and prognostic significance of CNS2 status and TLP positive findings.

\section{Author's contributions}

This work was carried out in collaboration between all authors. Author Hafiz MG designed the study, wrote the protocol, interpreted data and finally revised the paper. Author Jamal CY anchored field study and gathered initial data. Author Islam A performed preliminary data analysis. All authors read and approved the manuscript.

\section{Acknowledgements}

I express my sincere gratitude to the parents and or their relatives for their active cooperation regarding our data collection. I must express my gratitude to my eldest daughter Rezwana Binte Hafiz, Civil Engineer, for her sincere cooperation regarding statistical analysis and composing data. I would also give thanks to the nurses and staffs of the laboratory, Pediatric Hematology and Oncology, for their nice cooperation during this study.

\section{Conflicts of interest}

Author declares there are no conflicts of interest.

\section{Funding}

None.

\section{References}

1. Gupte S. 'Pediatric Oncology' in the short text book of Pediatrics, (9th edn), Jaypee brothers, Medical publishers (P) Ltd, New Delhi, India. 2001. p.438

2. Mannan MA. Paediatric Oncology Bangladesh-The long safari. Bangladesh J Child Health. 2003;27(2):3435.

3. Verman AJP. Acute lymphoblastic leukemia in children: Experiences in the West and East. Bangladesh J Child Health. 2003;27(2):16.

4. Eden OB. Management of acute lymphoblastic leukemia. Bangladesh $J$ Child Health. 2003;27(2):32-33.

5. Evans AE, Gilbert ES, Zandstra R. The increasing incidence of central nervous system leukemia in children. Cancer. 1970;26(2):404-409.

6. Reiter A, Schrappe M, Ludwig W-D, et al. Chemotherapy in 998 unselected childhood acute lymphoblastic leukemia patients. Results and conclusions of the multicenter trial ALL-BFM 86. Blood. 1994;84(9):3122-3133.

7. Smith M, Arthur D, Camitta B, et al. Uniform approach to risk classification and treatment assignment for children with acute lymphoblastic leukemia. J Clin Oncol . 1996;14(1):18-24.

8. Pui $\mathrm{CH}$, Crist W. Biology and treatment of acute lymphoblastic leukemia. J Pediatr. 1994;124(4):491-503.

9. Hammond D, Sather H, Nesbit M, et al. Analysis of prognostic factors in acute lymphoblastic leukemia. Med Pediatr Oncol . 1986;14(3):124-134.

10. Lilleyman JS, Eden OB. United Kingdom Medical Research Council Acute Lymphoblastic Leukemia (UKALL) Trials I-VIII: clinical features and results of treatment in four groups of children with adverse prognostic features. Med Pediatr Oncol. 1986;14(3):182-186.

11. Pui $\mathrm{CH}$, Mahmoud HH, Rivera GK, et al. Early intensification of intrathecal chemotherapy virtually eliminates central nervous system relapse in children with acute lymphoblastic leukemia. Blood. 1998;92(2):411-415.

12. Hill DE, Ciesielski KT, Sethre-Hofstad L, et al. Visual and verbal shortterm memory deficits in childhood leukemia survivors after intrathecal chemotherapy. J Pediatr Psychol . 1997;22(6):861-870.

13. Hilden JM, Dinndorf PA, Meerbaum SO, et al. CCG 1953: acute lymphoblastic leukemia in infants: analysis of prognostic factors: a report from the Children's Oncology Group. Blood. 2006; 108(2):441-451. 
14. Gajjar A, Harrison PL, Sandlund JT, et al. Traumatic lumbar puncture at diagnosis adversely affects outcome in childhood acute lymphoblastic leukemia. Blood. 2000;96(10):3381-3384.

15. Gilchrist G, Tubergen D, Sather H, et al. Low numbers of CSF blasts at diagnosis do not predict for the development of CNS leukemia in children with intermediate-risk acute lymphoblastic leukemia: A Children's Cancer Group report. J Clin Oncol. 1994;12(12):2594-2600.

16. Tubergen D, Cullen J, Boyett $\mathrm{J}$, et al. Blasts in CSF with a normal cell count do not justify alteration of therapy for acute lymphoblastic leukemia in remission: A Children's Cancer Group study. J Clin Oncol. 1994;12(12):273-278.

17. Mahmoud H, Rivera G, Hancock M, et al. Low leukocyte counts with blast cells in cerebrospinal fluid of children with newly diagnosed acute lymphoblastic leukemia. N Eng J Med. 1993; 329:314-319.

18. Bürger B, Zimmermann M, Mann G, et al. Diagnostic cerebrospinal fluid examination in children with acute lymphoblastic leukemia: significance of low leukocyte counts with blasts or traumatic lumbar puncture. J Clin Oncol. 2003;21(2):184-188.

19. Matloub Y, Bostrom BC, Hunger SP, et al. Escalating intravenous methotrexate improves event-free survival in children with standard-risk acute lymphoblastic leukemia: a report from the Children's Oncology Group. Blood. 2011;118(2):243-251.

20. Sirvent N, Suciu S, Rialland X, et al. Prognostic significance of the initial cerebro-spinal fluid (CSF) involvement of children with acute lymphoblastic leukaemia (ALL) treated without cranial irradiation: results of European Organization for Research and Treatment of Cancer (EORTC) Children Leukemia Group study 58881. Eur J Cancer. 2011;47(2):239-247.

21. Te Loo DM, Kamps WA, Van der Does-van den Berg A, et al. Prognostic significance of blasts in the cerebrospinal fluid without pleiocytosis or a traumatic lumbar puncture in children with acute lymphoblastic leukemia: experience of the Dutch Childhood Oncology Group. J Clin Oncol . 2006;24(15):2332-2336.

22. Pui CH, Campana D, Pei D, et al. Treating childhood acute lymphoblastic leukemia without cranial irradiation. $N$ Eng J Med. 2009;360(26):2730-2741.

23. Cherlow JM, Sather H, Steinherz P, et al. Craniospinal irradiation for acute lymphoblastic leukemia with central nervous system disease at diagnosis: a report from the Children's Cancer Group. Int J Radiat Oncol Biol Phys . 1996;36(1):19-27.

24. Mastrangelo R, Poplack D, Bleyer A, et al. Report and recommendations of the Rome workshop concerning poor-prognosis acute lymphoblastic leukemia in children: Biologic bases for staging, stratification and treatment. Med Pediatr Oncol. 1986;14(3):191-194.

25. Ribeiro RC, Pui $\mathrm{CH}$. The clinical and biologic features correlate of coagulopathy in children with acute leukemia. J Clin Oncol. 1986;4 (8):1212-1218.

26. Rivera GK, Raimondi SC, Behm FG, et al. Improved outcome in childhood acute lymphoblastic leukemia with reinforced early treatment and rotational combination chemotherapy. Lancet . 1991;337(8733):61-66.
27. Evans WE, Relling MV, Rodman JH, et al. Conventional compared with individualized chemotherapy for childhood acute lymphoblastic leukemia. N Engl J Med. 1998;338:499-505.

28. Cox DR. Regression models and life tables. $J R$ Stat Soc $B$. 1972;34(2):187-220.

29. Gilchrist G, Tubergen D, Coccia P, et al. Cerebrospinal fluid blasts (CSFB) on cytocentrifuge (CC) do not predict for central nervous system leukemia (CNSL) in children with intermediate-risk acute lymphoblastic leukemia (ALL): CCG-105. Proc Am Soc Clin Oncol 9:217-217 abstract. 1990.

30. Tubergen DG, Gilchrist GS, O'Brien RT, et al. Prevention of CNS disease in intermediate-risk acute lymphoblastic leukemia: comparison of cranial radiation and intrathecal methotrexate and the importance of systemic therapy: a Childrens Cancer Group report. J Clin Oncol. 1993; 11: 520-526.

31. Bleyer WA. Biology and pathogenesis of CNS leukemia. Am J Pediatr Hematol Oncol. 1989;11(1): 57-63.

32. Pinkel D, Woo S. Prevention and treatment of meningeal leukemia in children. Blood. 1994; 84(2):355-366.

33. Price RA, Johnson WW. The central nervous system in childhood leukemia. I: The arachnoid. Cancer. 1973;31(3):520-533.

34. Azzarelli B, Mirkin LD, Goheen M, et al. The leptomeningeal vein. A site of re-entry of leukemic cells into the systemic circulation. Cancer. 1984;54(7):1333-1343.

35. Pochedly C. Neurologic manifestations of leukemia. I: Symptoms due to increased CSF pressure and hemorrhage. II: Involvement of cranial nerves, hypothalamus, spinal cord, and peripheral neuropathy. In: Pochedly C (Ed.) Leukemia and Lymphoma in the Central Nervous System. Springfield, USA. 1977. P.3-40.

36. Rohlfing $\mathrm{MB}$, Barton $\mathrm{TK}$, Bigner $\mathrm{SH}$, et al. Contamination of cerebrospinal fluid with hematogenous blasts in patients with leukemia. Acta Cytol . 1981;25: 611-615.

37. Rubenstein JS, Yogev R. What represents pleocytosis in bloodcontaminated ("traumatic tap") cerebrospinal fluid in children? $J$ Pediatr. 1985;107(2): 249-251.

38. Osborne JP, Pizer B. Effect on the white cell count of contaminating cerebrospinal fluid with blood. Arch Dis Child. 1981;56(5): 400-401.

39. Macpherson CF, Lundblad LA. Conscious sedation of pediatric oncology patients for painful procedures: development and implementation of a clinical practice protocol. J Pediatr Oncol Nurs. 1997;14(1):33-42.

40. Marx CM, Stein J, Tyler MK, et al. Ketamine-midazolam versus meperidine-midazolam for painful procedures in pediatric oncology patients. J Clin Oncol . 1997;15(1):94-102.

41. Ferrari L, Barst S, Pratila M, et al. Anesthesia for diagnostic and therapeutic procedures in pediatric outpatients. Am J Pediatr Hematol Oncol . 1990;12(3):310-313. 